\title{
Hypothermic properties of dexmedetomidine provide neuroprotection in rats following cerebral ischemia-reperfusion injury
}

\author{
JIAN LU ${ }^{1,2}$, LI-JUN LIU ${ }^{1}$, JIAN-LIANG ZHU ${ }^{1}$, YI SHEN ${ }^{2}$, ZHI-WEI ZHUANG ${ }^{2}$ and CHANG-LAI ZHU ${ }^{3}$ \\ ${ }^{1}$ Department of Emergency and Critical Care Medicine, The Second Affiliated Hospital of Soochow University, \\ Suzhou, Jiangsu 215007; ${ }^{2}$ Department of Emergency and Critical Care Medicine, The Affiliated Suzhou Hospital of \\ Nanjing Medical University, Suzhou Municipal Hospital, Suzhou, Jiangsu 215002; ${ }^{3}$ Key Laboratory of \\ Neuroregeneration, Nantong Medical College of Nantong University, Nantong, Jiangsu 226200, P.R. China
}

Received September 10, 2018; Accepted April 18, 2019

DOI: $10.3892 /$ etm.2019.7613

\begin{abstract}
Dexmedetomidine (Dex) is a sedative and analgesic agent that is widely administered to patients admitted to the intensive care unit, and has been demonstrated to result in hypothermia. Many patients have been revealed to benefit from therapeutic hypothermia, which can mitigate cerebral ischemia/reperfusion (I/R) injury following successful cardiopulmonary resuscitation. However, studies investigating the efficacy of Dex in I/R treatment is lacking. The present study aimed to investigate the efficacy of Dex in mitigating neuronal damage, and to determine the possible mechanism of its effects in a rat model of cardiac arrest (CA). CA was induced in Sprague-Dawley rats by asphyxiation for $5 \mathrm{~min}$. Following successful resuscitation, the surviving rats were randomly divided into two treatment groups; one group was intraperitoneally administered with Dex (D group), whereas the control group was treated with normal saline ( $\mathrm{N}$ group). Critical parameters, including core temperature and blood pressure were monitored following return of spontaneous circulation (ROSC). Arterial blood samples were collected at $10 \mathrm{~min}$ after surgery (baseline) 30 and 120 min post-ROSC; and neurological deficit scores (NDS) of the rats were taken 12 or $24 \mathrm{~h}$ after ROSC prior to euthanasia. The hippocampal tissue was then removed for analysis by histology, electron microscopy and western blotting. Rats in the D group exhibited a lower core temperature and higher NDS scores compared with the $\mathrm{N}$ group $(\mathrm{P}<0.05)$. In addition, Dex injection resulted in reduced expression of apoptotic and autophagy-associated factors in the hippocampus $(\mathrm{P}<0.05)$. Dex treatment induced hypothermia and improved
\end{abstract}

Correspondence to: Dr Li-Jun Liu, Department of Emergency and Critical Care Medicine, The Second Affiliated Hospital of Soochow University, 1055 Sanxiang Road, Suzhou, Jiangsu 215007, P.R. China E-mail: 1ijunliusz@sina.com

Key words: dexmedetomidine, hypothermia, cardiopulmonary resuscitation, hippocampus, apoptosis, autophagy neurological function in rats after ROSC following resuscitation from CA by inhibiting neuronal apoptosis and reducing autophagy, which suggested that Dex may be a potential therapy option for patients with CA.

\section{Introduction}

Mortality and morbidity rates as a result of cardiac arrest (CA) remain high despite steady advances in therapeutic approaches over the past decades (1). Post-resuscitation neurological dysfunction is a major cause of mortality in patients following successful cardiopulmonary resuscitation (CPR) $(2,3)$. Therefore, it is vital to research strategies to protect neurological function in patients following CA.

Although CA can lead to ischemia/reperfusion (I/R) injury in every organ in the body, it is especially detrimental to the brain and the heart. The principal pathological cause of organ damage is excessive apoptosis and autophagy (4,5), the extent of which has been found to correlate with poor neurological prognosis (6-8). A number of studies have demonstrated that hypothermia can attenuate neurological damage following I/R injury by reducing neural apoptosis and preventing autophagy overactivation $(4,9,10)$. Therefore, it has been recommended as a potential treatment in patients who were successfully resuscitated following CA (11).

Apoptosis is a form of cell death that is mediated by several proteins, including Bax, Bcl-2, $70 \mathrm{kDa}$ heat shock protein (HSP70) and cysteinyl aspartate specific proteinase (Caspase-3) $(12,13)$. Bax and Bcl-2 are members of the Bcl-2 family of molecular protein factors that can reflect the extent of cellular apoptosis (14); whereas HSP70 is a highly conserved molecular chaperone that serves an important role in cellular adaptation to stress, a requisite for cell survival (15). Lastly, Caspase- 3 is a commonly probed apoptosis marker, as it takes part in the execution-phase of the apoptotic pathway $(13,16,17)$.

Autophagy is the primary mechanism by which cytosolic proteins and organelles are degraded (18). However, excessive autophagy activation in neurocytes damaged by $\mathrm{I} / \mathrm{R}$ can lead to non-apoptotic cell death $(19,20)$, which is called autoghagic programmed cell death. Beclin1 activates critical 
steps in autophagy, including the formation and maturation of autophagosomes $(21,22)$. In addition, Bax and Bcl-2 have also been demonstrated to regulate autophagy (12). In fact, it has been well documented that the crosstalk between autophagy and apoptosis pathways is mediated at least in part by the functional and structural interaction between Beclin1 and Bcl-2 (23).

Dexmedetomidine (Dex) is a highly selective $\alpha_{2}$-adrenergic receptor agonist that is commonly used as a sedative and analgesic in the intensive care unit (24); it has been reported to display synergistic effects with hypothermia $(25,26)$. Consequently, based on previous observations that hypothermia can alleviate neuronal damage following CA, it was hypothesized in the present study that Dex administration after CA may result in improved neural function and reduced apoptosis and autophagy. The present study also aimed to investigate the possible mechanism by which Dex improves cognitive function.

\section{Materials and methods}

Animal preparation and Experimental protocol. All operations and euthanasia were performed under anesthesia induced by an intraperitoneal (i.p.) injection of $3.6 \%$ chloral hydrate solution (3.6 g chloral hydrate crystal diluted with $100 \mathrm{ml}$ distilled water) at a concentration of $360 \mathrm{mg} / \mathrm{kg}$, and the best efforts were made to minimize animal suffering.

A total of 72, male Sprague-Dawley rats [age, 17 and 18 weeks; weight, 430 \pm 45 g; JOINN Laboratories (China) Co., Ltd.] were recruited successfully. They were provided food and water ad libitum, and housed at $25^{\circ} \mathrm{C}$, with freshly ventilator delivered atmosphere and a $12 \mathrm{~h}$ light/dark cycle. The procedure of animal CA and CPR were performed as described previously (4). Endotracheal intubation was performed prior to left/right femoral arteriovenous catheterization. Animals were ventilated with a volume-controlled ventilator (Institute of Cardiopulmonary Cerebral Resuscitation, Guangdong, China), with a tidal volume of $6 \mathrm{ml} / \mathrm{kg}$, a fraction of inspired oxygen $\left(\mathrm{FiO}_{2}\right)$ of 0.21 , and a ventilation rate of 100 breaths per min. Continuous arterial blood pressure was monitored via an intra-arterial pressure sensor suite (Philips Medical Systems, Inc.) and an electrocardiogram (Anhui Zhenghua Biological Instrument Equipment Co., Ltd.). Baseline arterial blood gas analysis was performed 10 min after the surgical procedure. An equal volume $(0.5 \mathrm{ml})$ of normal saline was supplemented into the venous catheter immediately after arterial blood collection. CA was induced in the animals by reducing mechanical ventilation and occluding the tracheal catheter. For CPR, external chest compression $(250 \mathrm{bpm})$ and mechanical ventilation $(6 \mathrm{ml} / \mathrm{kg}$ and $100 \mathrm{bpm})$ was performed after a period of $5 \mathrm{~min}$ under systolic blood pressure $<25 \mathrm{mmHg}$. After $10 \mathrm{sec}$ of CPR all rats were administered a bolus injection of epinephrine $(10 \mu \mathrm{g} / \mathrm{kg}$, diluted to $0.5 \mathrm{ml})$ in the right femoral vein. In addition to epinephrine, before CPR execution, one group of rats received an i.p. injection of Dex (50 $\mu \mathrm{g} / \mathrm{kg}(27)$, diluted to $0.5 \mathrm{ml}$; D group) whereas another group received an i.p. injection of $0.9 \%$ normal saline ( $0.5 \mathrm{ml}$; $\mathrm{N}$ group). ROSC achievement was defined as the presence of an autonomic cardiac rhythm and a mean arterial blood pressure $>60 \mathrm{mmHg}$. At this point, chest compressions were stopped, $\mathrm{a} \mathrm{FiO}_{2}$ of $100 \%$ was maintained for 15 min after ROSC, decreased to $45 \%$ for a further $15 \mathrm{~min}$ and then decreased to $21 \%$ until the animal exhibited spontaneous respirations. Resuscitation procedures were terminated if animals were unresponsive to CPR for $10 \mathrm{~min}$. After $2 \mathrm{~h}$ of monitoring animal vital signs, rats returned to consciousness, were placed into a thermostabilized $\left(25^{\circ} \mathrm{C}\right)$ cage for rehabilitation and euthanized either $12(\mathrm{~N}-12, \mathrm{n}=15$; $\mathrm{D}-12, \mathrm{n}=15)$ or $24 \mathrm{~h}(\mathrm{~N}-24, \mathrm{n}=14 ; \mathrm{D}-24, \mathrm{n}=13)$ later. A total of 9 rats died during the recovery period in the cage due to airway edema and sputum obstruction, which was certified following autopsy. Rats belonging to the blank control group $(B C ; n=6)$ received identical surgical and anesthetic treatments as the experimental rats with the exception of CA and CPR.

Critical parameters, including core (rectal) temperature and blood pressure were monitored continuously for $120 \mathrm{~min}$ (timed from the first sign of ROSC) after CPR until the rats regained consciousness. Within the same timeframe, arterial blood gas was also analyzed 30 and $120 \mathrm{~min}$ after CPR.

Neurological deficit scores (NDS). NDS is a common procedure for evaluating neurological dysfunction by using a hierarchical scoring system based on respiratory pattern, consciousness, sensory and motor function and behavioral responses (4). Every animal was evaluated 12 or $24 \mathrm{~h}$ following return of spontaneous circulation (ROSC). The NDS items were collected by investigators that have been specially trained, who were blinded to the experimental groups. Individual scores were subsequently combined to produce the final NDS score. A score of 80 is considered normal whereas a score of zero is considered brain dead (28).

Collection of rat hippocampal tissues. After the NDS scores were obtained, rats were anesthetized using a combination of an i.p. injection of $3.6 \%$ chloral hydrate solution at $360 \mathrm{mg} / \mathrm{kg}$ and an intracardial injection of $500 \mathrm{mg} / \mathrm{kg} 10 \%$ potassium chloride solution. The hippocampus was rapidly removed from the cerebrum of the rat. The left half was snap frozen using liquid nitrogen before storage at $-80^{\circ} \mathrm{C}$ to be used later for western blot analysis of Bcl-2, Bax, HSP70 and Beclin1 protein expression. The right half of the hippocampus was fixed in $4 \%$ paraformaldehyde for $24 \mathrm{~h}$ at $4^{\circ} \mathrm{C}$ and subsequently embedded in paraffin for analysis using histology techniques.

Western blot analysis of Bcl-2, Bax, HSP70 and Beclin1 expression in the rat hippocampus. Hippocampal tissue protein was extracted with $400 \mu 1$ RIPA and $4 \mu 1$ PMSF (Beyotime Institute of Biotechnology). Samples were then homogenized on the ice and centrifuged in a refrigerated centrifuge $(12,000 \times \mathrm{g}$ for $10 \mathrm{~min}$ at $4^{\circ} \mathrm{C}$ ). Protein concentration in the supernatant from hippocampal homogenate was determined using a Bicinchoninic Acid Assay kit (Beyotime Institute of Biotechnology). A total of $50 \mu \mathrm{g}$ of protein obtained from the hippocampus were separated by 10 or $12 \%$ SDS-PAGE before transferal to a PVDF membrane. Each membrane was blocked in 5\% non-fat milk diluted in TBS supplemented with $0.1 \%$ Tween-20 (TBST) for $1 \mathrm{~h}$ at $4^{\circ} \mathrm{C}$. The membranes were then incubated overnight at $4^{\circ} \mathrm{C}$ with primary antibodies against Bcl-2 (1:1,000; cat. no. ab196495), Bax (1:1,000; cat. no. ab32503), HSP70 (1:1,000; cat. no. ab137680), Beclin1 (1:1,000; cat. no. ab62557) or Glyceraldehyde-3-phosphate dehydrogenase (GAPDH; 1:800; 
Table I. Cardiac arrest time and associated resuscitation data.

\begin{tabular}{|c|c|c|c|c|c|c|}
\hline Group & $\begin{array}{l}\text { Valid } \\
\text { rats (n) }\end{array}$ & $\begin{array}{c}\text { Time of } \\
\text { operation }(\mathrm{sec})\end{array}$ & $\begin{array}{c}\text { Time of } \\
\text { stabilization for } \\
\text { baseline }(\mathrm{sec})\end{array}$ & $\begin{array}{l}\text { Time from } \\
\text { asphyxia to } \mathrm{SBP} \\
<25 \mathrm{mmHg}(\mathrm{sec})\end{array}$ & $\begin{array}{c}\text { Time of SBP } \\
<25 \mathrm{mmHg}(\mathrm{sec})\end{array}$ & $\begin{array}{l}\text { Time from } \\
\text { resuscitation } \\
\text { to } \operatorname{ROSC}(\mathrm{sec})\end{array}$ \\
\hline $\mathrm{N}$ & 29 & $485.61 \pm 62.47$ & $589.24 \pm 15.72$ & $221.26 \pm 36.43$ & $272.34 \pm 36.75$ & $86.41 \pm 32.67$ \\
\hline $\mathrm{D}$ & 28 & $505.73 \pm 58.32$ & $596.12 \pm 12.67$ & $234.82 \pm 32.48$ & $268.28 \pm 38.74$ & $89.64 \pm 29.53$ \\
\hline
\end{tabular}

D, dexmedetomidine group; N, normal saline group; ROSC, return of spontaneous circulation; SBP, systolic blood pressure.

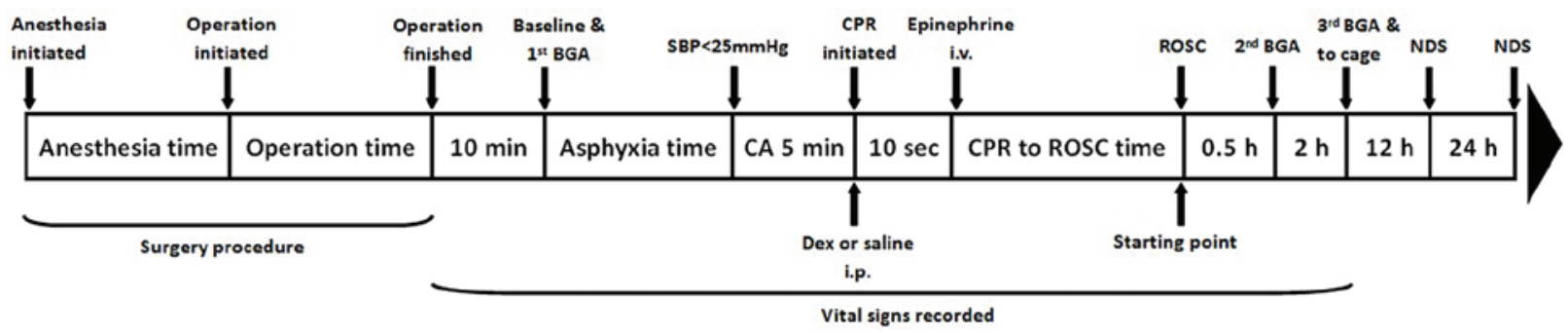

Figure 1. Experimental scheme for animal treatment. SBP, systolic blood pressure; BGA, blood gas analysis; NDS, neurological deficit scores; CA, cardiac arrest; CPR, cardiopulmonary resuscitation; ROSC, return of spontaneous circulation.

cat. no. ab9485); all primary antibodies applied for western blot analysis were purchased from Abcam. The following day, the membranes were rinsed with TBS-T and subsequently incubated with a biotin-conjugated goat anti-rabbit IgG secondary antibody (1:5,000; cat. no. ab222773; Abcam) at room temperature for $1 \mathrm{~h}$. Protein bands were visualized using an ECL reagent (Biological Industries Israel Beit Haemek Ltd.). Densitometric analysis was performed using Quantity One 1-D analysis software (version 4.6.9; Bio-Rad Laboratories, Inc.); the protein levels of Bcl-2, Bax, HSP70 and Beclin1 were all normalized to GAPDH.

Immunohistochemistry analysis of Caspase-3. Paraffin-embedded hippocampal tissue was cut into $5 \mu \mathrm{m}$ sections using a sledge microtome. The tissue was deparaffinized using xylene and rehydrated in a series of descending concentrations of ethanol. Antigen retrieval was completed by boiling in citric acid buffer $(10 \mathrm{mM}, \mathrm{pH} 6.0)$ at $95^{\circ} \mathrm{C}$ for $15 \mathrm{~min}$. $0.5 \%$ Triton X-100 (Beyotime Institute of Biotechnology) was used for membrane permeabilization. The slides were blocked at $37^{\circ} \mathrm{C}$ for $2 \mathrm{~h}$ in $5 \%$ bovine serum albumin (BSA; Beyotime Institute of Biotechnology) diluted in TBS and subsequently incubated in anti-caspase-3 antibody (1:100; cat. no. ab4051; Abcam) diluted in TBS supplemented with 5\% BSA overnight at $4^{\circ} \mathrm{C}$. The following day, the sections were rinsed in TBS prior to incubation with a biotin-conjugated goat anti-rabbit secondary antibody (1:500; cat. no. ab222773) for $1 \mathrm{~h}$ at $37^{\circ} \mathrm{C}$. The secondary antibody was washed off using TBS and the slides were incubated under $37^{\circ} \mathrm{C}$ for $5-10 \mathrm{~min}$ in a solution containing $0.02 \%$ diaminobenzidine (DAB) and $0.01 \% \mathrm{H}_{2} \mathrm{O}_{2}$ and then counterstained using hematoxylin and eosin $(\mathrm{H} \& \mathrm{E})$ at $37^{\circ} \mathrm{C}$ for $10 \mathrm{~min}$. The slides were imaged using a light microscope (Olympus BX53; Olympus Corporation). Cells exhibiting brown staining were considered Caspase- 3 positive and were counted using the Leica software analysis system (Q550CW;
Leica Microsystems, Inc). Each group was observed in four different fields of view; 100 cells were observed in the field of vision and then caspase-3 positive cells were calculated. Counting was performed by a specialized pathologist who was blind to the experimental groups.

Transmission electron microscopy (TEM). The paraffinembedded tissues were also used for histological analysis using TEM. This tissue was sliced using a microtome into ultra-thin sections $(\sim 40-50 \mathrm{~nm})$. The sections were then stained with $2.0 \%(\mathrm{w} / \mathrm{v})$ lead citrate at $4^{\circ} \mathrm{C}$ for $10 \mathrm{~min}$ and evaluated using a Hitachi H-600 transmission electron microscope (Hitachi, Ltd.) in a blinded fashion. For every section, four different TEM micrographs each representing independent fields of view were analyzed.

Statistical analysis. All experimental data are presented as the mean \pm SD. A t-test was used to compare the means between the two groups. Multiple-comparison analyses performed in this study were confirmed using Levene's test for the equality of variances. A one-way ANOVA was applied to assess holistic differences between groups for each variable, and Bonferroni post-hoc test was used for multiple comparisons. The software package used to perform the analysis was IBM SPSS Statistics 22.0 (IBM Corp.). $\mathrm{P}<0.05$ was considered to indicate a statistically significant difference.

\section{Results}

Animal hemodynamic, physiological, and resuscitation data. The experimental scheme of animal treatment for the present study is detailed in Fig. 1. No statistically significant differences were observed in operation, hemodynamic stabilization, asphyxia, CA and CPR time between the resuscitation groups (Table I). In addition, no significant differences were 


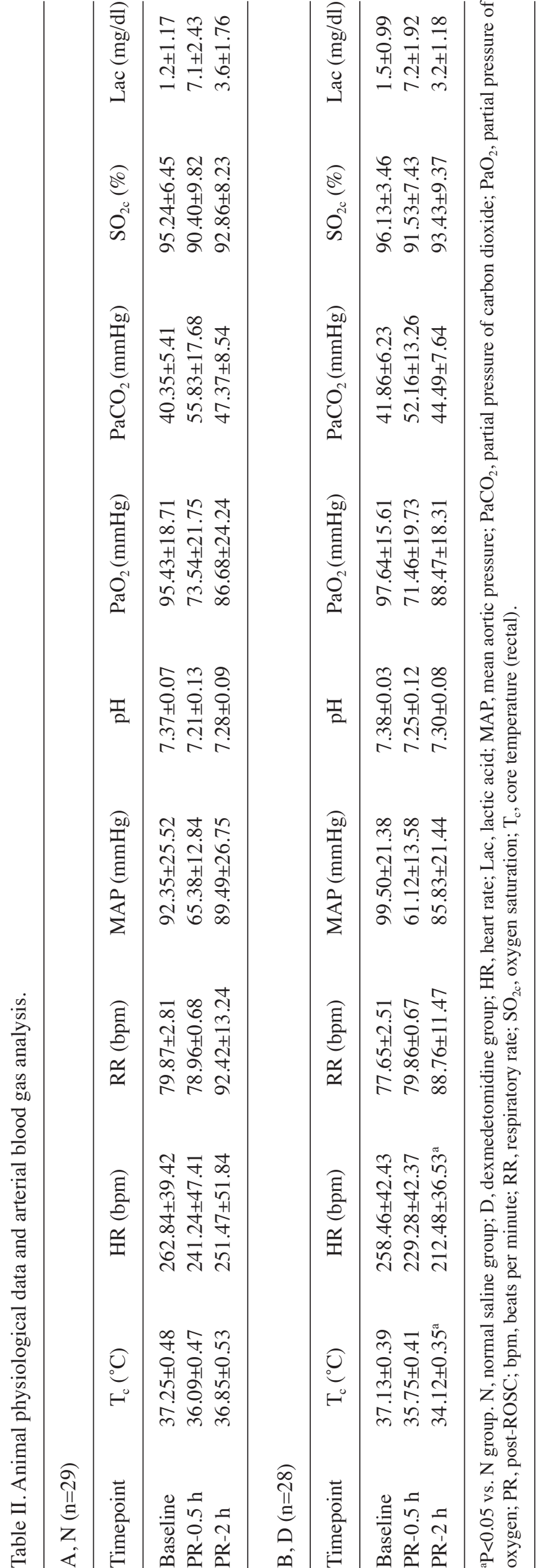

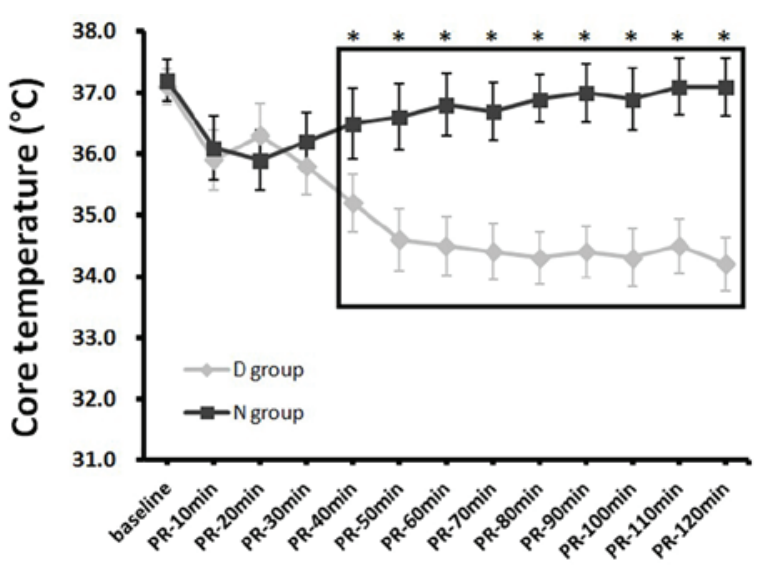

Figure 2. Measurement of rat core (rectal) temperature from baseline up to $120 \mathrm{~min}$ post-ROSC. The core temperatures between the two groups exhibited statistically significant differences from $40 \mathrm{~min}$ onwards after ROSC (black box). ${ }^{*} \mathrm{P}<0.05$ vs. N group. D group, dexmedetomidine treatment group; $\mathrm{N}$ group, normal saline group; PR, post-ROSC.

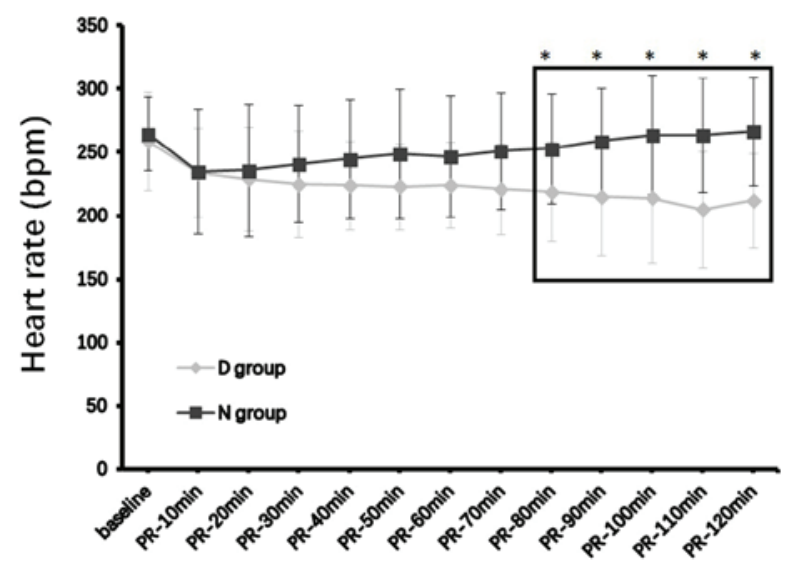

Figure 3. Heart rate measurements in rats from baseline to $120 \mathrm{~min}$ post-ROSC. The heart rates between the two groups exhibited statistically significant differences from 80 min after ROSC (black box). ${ }^{*} \mathrm{P}<0.05$ vs. $\mathrm{N}$ group. bpm, beat per minute; D group, dexmedetomidine group; $\mathrm{N}$ group, normal saline group; PR, post-ROSC; ROSC, return of spontaneous circulation.

identified between the groups with regards to the respiration rate, mean aortic blood pressure, arterial blood $\mathrm{pH}$, oxygen and carbon dioxide partial pressure, oxygen saturation and lactic acid during baseline, at either $30 \mathrm{~min}$ or $2 \mathrm{~h}$ after ROSC (Table II). Core body temperatures and heart rates in the D group decreased over time, and began to exhibit statistically significant reductions compared with the $\mathrm{N}$ group from 40 and $80 \mathrm{~min}$ post-ROSC, respectively, up to post-ROSC $120 \mathrm{~min}$ when the analyses ended (both $\mathrm{P}<0.05$; Figs. 2 and 3; Table II).

Neurological outcome. NDS was determined before the rats were euthanized at 12 or $24 \mathrm{~h}$ post-ROSC. The mean NDS value of rats in the $\mathrm{N}-12(43.5 \pm 5.7)$ and $\mathrm{N}-24(50.6 \pm 5.1)$ groups was significantly lower compared with the $\mathrm{BC}$ group $(80.0 \pm 0.0$; $\mathrm{P}<0.05$; Fig. 4). However, post-ROSC NDS was higher in the D-12 (56.2 \pm 6.1$)$ and D-24 (63.4 \pm 4.9$)$ groups compared with their respective $\mathrm{N}-12$ and $\mathrm{N}-24$ timepoints $(\mathrm{P}<0.05$; Fig. 4). These findings suggested that the use of Dex post-ROSC in rats improved neurological outcomes after CA compared with untreated animals. 


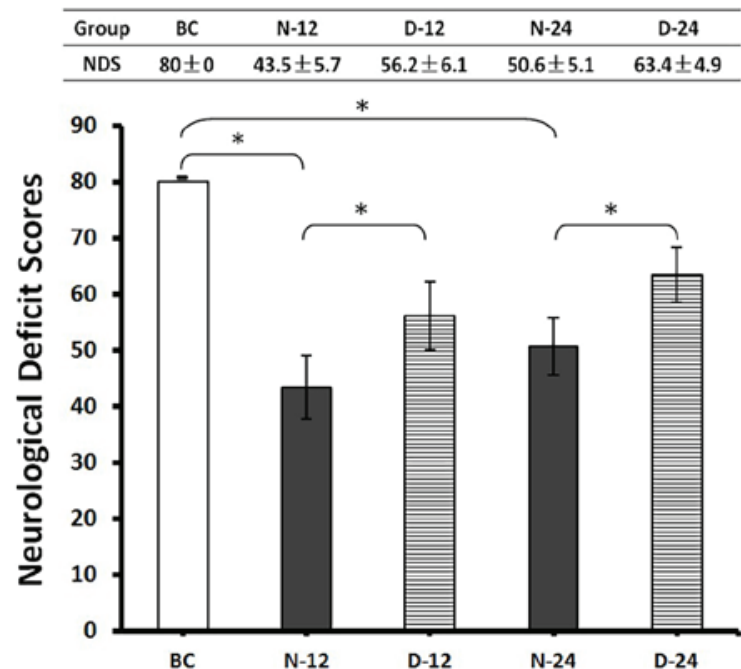

Figure 4. Rat neurological deficit scores. NDSs were evaluated 12 and $24 \mathrm{~h}$ after ROSC in the presence of normal saline or dexmedetomidine. ${ }^{*} \mathrm{P}<0.05$. ROSC, return of spontaneous circulation; BC, blank control group; D-12, dexmedetomidine group $12 \mathrm{~h}$ post-ROSC; D-24, dexmedetomidine group $24 \mathrm{~h}$ post-ROSC; $\mathrm{N}-12$, normal saline group $12 \mathrm{~h}$ post-ROSC; N-24, normal saline group $24 \mathrm{~h}$ post-ROSC.

Intracellular Bax, Bcl-2, HSP70 and Beclin1 protein expression levels in the hippocampus. Bax expression was found to be greater in the N-12 $(0.024 \pm 0.002)$ and N-24 $(0.022 \pm 0.006)$ groups post-ROSC compared with the $\mathrm{BC}$ group $(0.003 \pm 0.000)$ $(\mathrm{P}<0.05$; Fig. 5A). In addition, Bax expression was significantly lower in the D-12 $(0.017 \pm 0.001)$ and D-24 $(0.006 \pm 0.004)$ groups post-ROSC compared with the respective N-12 and N-24 timepoints $(\mathrm{P}<0.05$; Fig. 5A). Bcl-2 expression was significantly reduced in the $\mathrm{N}-12(0.019 \pm 0.001)$ and $\mathrm{N}-24(0.014 \pm 0.001)$ groups post-ROSC compared with the BC group $(0.022 \pm 0.000)$ $(\mathrm{P}<0.05$; Fig. 5B). However, Bcl-2 expression was significantly higher in the D-12 (0.025 \pm 0.001$)$ and D-24 (0.024 \pm 0.006$)$ groups post-ROSC compared with their respective $\mathrm{N}-12$ and $\mathrm{N}-24$ timepoints $(\mathrm{P}<0.05$; Fig. 5B). HSP70 expression was higher in the N-12 $(0.45 \pm 0.07)$ and N-24 $(0.41 \pm 0.05)$ groups post-ROSC compared with the BC group $(0.32 \pm 0.00)(\mathrm{P}<0.05$; Fig. $5 \mathrm{C})$. In addition, HSP70 expression was higher in the D-12 (0.56 \pm 0.10$)$ and D-24 (0.54 \pm 0.06$)$ groups compared with the respective $\mathrm{N}-12$ and $\mathrm{N}-24$ timepoints $(\mathrm{P}<0.05$; Fig. 5C). Beclin1 expression was higher in the N-12 $(0.43 \pm 0.05)$ and N-24 $(0.39 \pm 0.04)$ groups post-ROSC compared with the $\mathrm{BC}$ group $(0.15 \pm 0.00)(\mathrm{P}<0.05$; Fig. 5D). However, Beclin1 expression was lower in the D-12 $(0.31 \pm 0.06)$ and D-24 (0.25 \pm 0.03$)$ groups post-ROSC compared with their respective $\mathrm{N}-12$ and $\mathrm{N}-24$ timepoints $(\mathrm{P}<0.05$; Fig. 5D).

Measurement of apoptosis in the rat hippocampus. Neuronal apoptosis was determined by counting the number of Caspase-3-positive staining cells located in the CA1 region of the hippocampus. The intense staining of surrounding tissues was impacted by the time of DBA coloration and the brown staining inside cells was defined as apoptosis-positive. The number of apoptotic neurons were demonstrated to be more abundant in the $\mathrm{N}-24$ group compared with the BC group $(\mathrm{P}<0.05$; Fig. 6). However, fewer hippocampal neurons were stained positive for Caspase-3 in the D-24 group compared with $\mathrm{N}-24$ group $(\mathrm{P}<0.05$; Fig. 6$)$.
Histological and ultrastructural analysis in the rat hippocampus. Histological analysis of hippocampal tissues from the three groups was carried out using H\&E staining $24 \mathrm{~h}$ post-ROSC; following which the nuclei of cells located in the CA1 region of the hippocampus were examined. Compared with the distinct shape and content of neurocytes from BC group (Fig. 7A; white arrows), cells from the N-24 group exhibited faint nuclear shapes with compact cytoplasm (Fig. 7B; yellow arrows), which indicated that hippocampal neurons were damaged after I/R. Cells from the D-24 group hippocampus displayed visible nuclei and distinct cytoplasm (Fig. 7C; green arrows) compared with $\mathrm{N}$ group, which suggested that Dex treatment reduced neural damage following I/R.

According to the TEM micrographs, the extent of nuclear ultrastructural damage in the hippocampus was less pronounced in the D-24 group compared with the N-24 group. The BC group exhibited a nucleus that appeared normal with an intact nuclear membrane (Fig. 8A), whereas that in the $\mathrm{N}$ group was markedly damaged displaying chromatin margination and distorted mitochondria (Fig. 8B). In the $\mathrm{D}$ group, these features of damage were less visible (Fig. 8C). In addition, autophagolysosomes (Fig. 8B; black arrows) and heteromorphic mitochondria (Fig. 8B; red arrows) could be observed in the $\mathrm{N}$ group. However, little to none of these features were identified in the $\mathrm{D}$ group.

\section{Discussion}

The present study found that Dex administration may induce mild hypothermia, slow down heart rate, attenuate apoptosis of neurocytes and improve neurological function after ROSC following resuscitation from CA. This is supported by histological and ultrastructural observations that Dex reduced damage in the rat hippocampal tissue. Mechanistically, the protective effect of Dex was found to be associated with the downregulation of pro-apoptotic proteins Bax and Caspase-3 with a concomitant upregulation in anti-apoptotic proteins Bcl-2 and HSP70. This is coupled with the finding that the expression of autophagic protein Beclin1 was downregulated in Dex-treated hippocampal tissue.

Dex is an $\alpha-2 \mathrm{~A}$ adrenergic receptor agonist commonly administered to patients as a sedative and analgesic (29). Previous studies have reported Dex to be neuroprotective when combined with hypothermia $(30,31)$. However, few studies have assessed the association between Dex and hypothermia, making the mechanism of this process unclear. Lähdesmäki et al (32) determined that the hypothermic effect of Dex may be abolished if $\alpha-2 \mathrm{~A}$ adrenergic receptor genes were knocked out in mice, which indicated that the activation of $\alpha-2 \mathrm{~A}$ adrenergic receptors, induced by Dex, may inhibit neuronal firing and the release of monoamine neurotransmitters associated with locomotor activity and body temperature. The present study found that the core temperature and heart rate in the group of rats treated with Dex was lower when compared with the saline group in the same post-ROSC environment; which corresponded well with the results from other studies. For example, one previous report suggested that Dex can sustain hypothermia by suppressing muscle shivering (33). It was hypothesized that this effect is caused by the inhibition of neurotransmitter release from sympathetic nerves located 

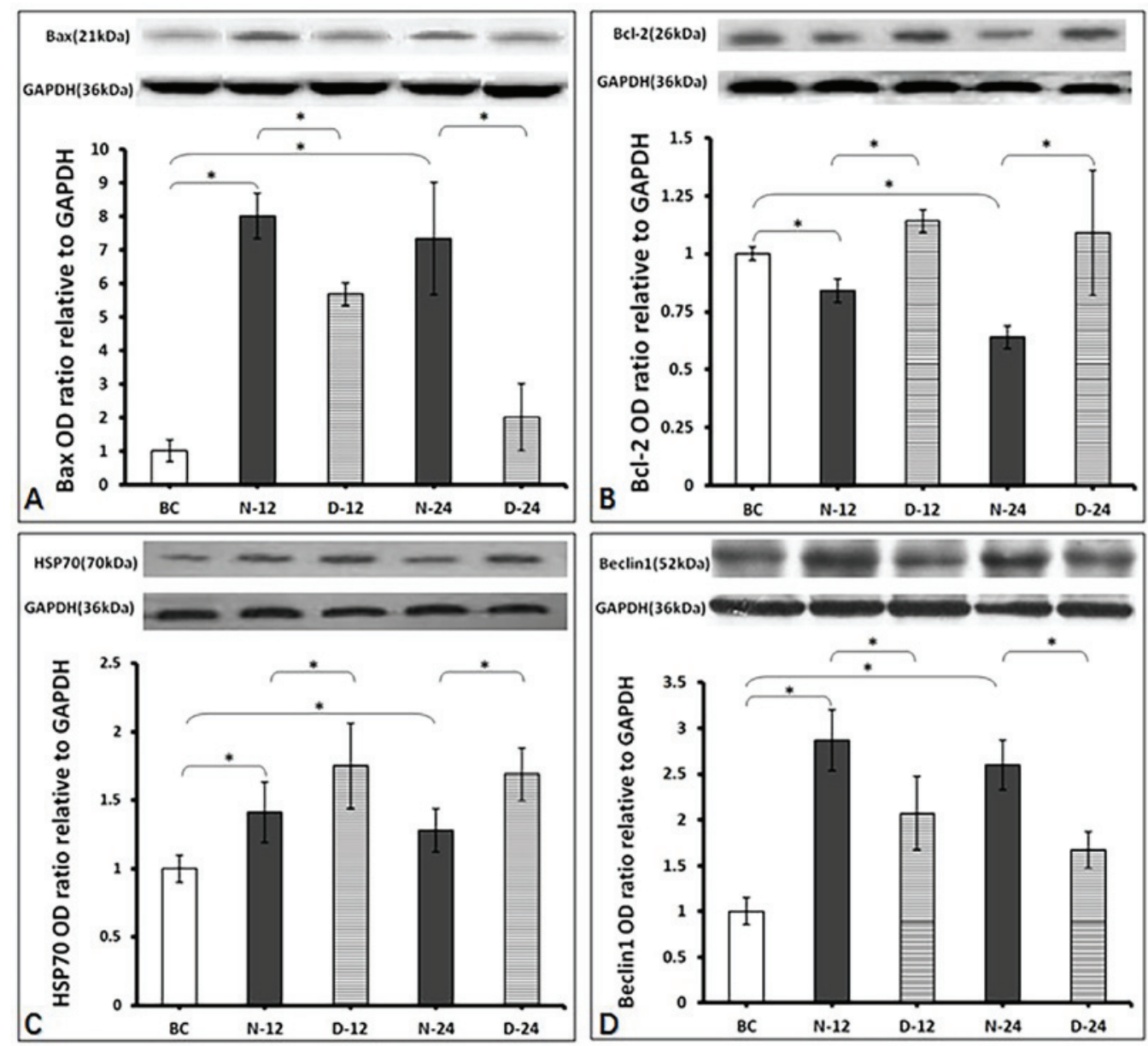

Figure 5. Western blotting results. All data values were normalized to the values of BC group (as level ' 1 '). (A) Measurement of Bax protein. The expression levels were in the rat hippocampus from each treatment group 12 and $24 \mathrm{~h}$ after ROSC, in the presence of normal saline or dexmedetomidine. "P $<0.05$. $\mathrm{BC}$, blank control group; D-12, dexmedetomidine group $12 \mathrm{~h}$ post-ROSC; D-24, dexmedetomidine group $24 \mathrm{~h}$ post-ROSC; N-12, normal saline group $12 \mathrm{~h}$ post-ROSC; N-24, normal saline group $24 \mathrm{~h}$ post-ROSC; ROSC, return of spontaneous circulation. (B) Measurement of Bcl-2 protein. The expression levels were in the rat hippocampus from each treatment group 12 and $24 \mathrm{~h}$ after ROSC, in the presence of normal saline or dexmedetomidine. ${ }^{*} \mathrm{P}<0.05$. $\mathrm{BC}$, blank control group; D-12, dexmedetomidine group $12 \mathrm{~h}$ post-ROSC; D-24, dexmedetomidine group $24 \mathrm{~h}$ post-ROSC; N-12, normal saline group $12 \mathrm{~h}$ post-ROSC; $\mathrm{N}-24$, normal saline group $24 \mathrm{~h}$ post-ROSC; ROSC, return of spontaneous circulation. (C) Measurement of HSP70 protein. The expression levels were in the rat hippocampus from each treatment group 12 and $24 \mathrm{~h}$ after ROSC, in the presence of normal saline or dexmedetomidine. ${ }^{*} \mathrm{P}<0.05$. BC, blank control group; D-12, dexmedetomidine group $12 \mathrm{~h}$ post-ROSC; D-24, dexmedetomidine group $24 \mathrm{~h}$ post-ROSC; HSP70, heat shock protein $70 \mathrm{kDa}$; N-12, normal saline group post-ROSC $12 \mathrm{~h}$; N-24, normal saline group $24 \mathrm{~h}$ post-ROSC; ROSC, return of spontaneous circulation. (D) Measurement of Beclin1 protein. The expression levels were in the rat hippocampus from each treatment group 12 and $24 \mathrm{~h}$ after ROSC, in the presence of normal saline or dexmedetomidine. ${ }^{*} \mathrm{P}<0.05$. BC, blank control group; D-12, dexmedetomidine group $12 \mathrm{~h}$ post-ROSC; D-24, dexmedetomidine group $24 \mathrm{~h}$ post-ROSC; N-12, normal saline group $12 \mathrm{~h}$ post-ROSC; $\mathrm{N}-24$, normal saline group $24 \mathrm{~h}$ post-ROSC; ROSC, return of spontaneous circulation.

within the skeletal muscles by blocking temperature-sensitive vanilloid-type transient receptor potential ion channels (34). In addition, in the sinoatrial node, Dex has been demonstrated to activate cardiac $\mathrm{Ca}^{2+}$-sensitive potassium ion channels in pacemaker cells during repolarization (35), causing a reduction in heart rate.

It was demonstrated in our previous study that mild hypothermia can reduce the level of lactic acid in the blood, alleviate neurocyte apoptosis and improve neurological function in rats that were resuscitated from CA (4). Sato et al (30) found in a rat model of focal cerebral ischemia that short-term neurological outcomes could be improved by a combination of Dex and hypothermia. In the current study, Dex induced hypothermia and improved neurological function in rats after ROSC following CA. However, no difference was found in lactic acid levels between the two groups; this may be due to the collection of arterial blood in $2 \mathrm{~h}$ intervals, which did not provide enough time for lactic acid metabolism and excretion. Another possibility was that the duration and severity of hypothermia was not adequate for the elimination of lactic acid, or that Dex may enhance the risk of metabolic acidosis (36).

Zhang et al (37) recently reported that HSP70 conferred cellular protection against ischemic insults and possessed potent anti-apoptotic properties in hippocampal neurons using a traumatic brain injury rat model. To further understand the role of apoptosis and autophagy in neurons after $\mathrm{I} / \mathrm{R}$, protein expression levels of Bax, Bcl-2, Caspase-3 and Beclin1 were analyzed in the present study. Bax, Caspase-3 and Beclin1 expression increased after ROSC, suggesting that apoptosis and autophagy were activated by I/R in the hippocampus. However, Dex administration inhibited this increase, subsequently improving neurological function. Therefore, it is possible that Dex may preserve neurological function by reducing neural apoptosis and autophagy following I/R 

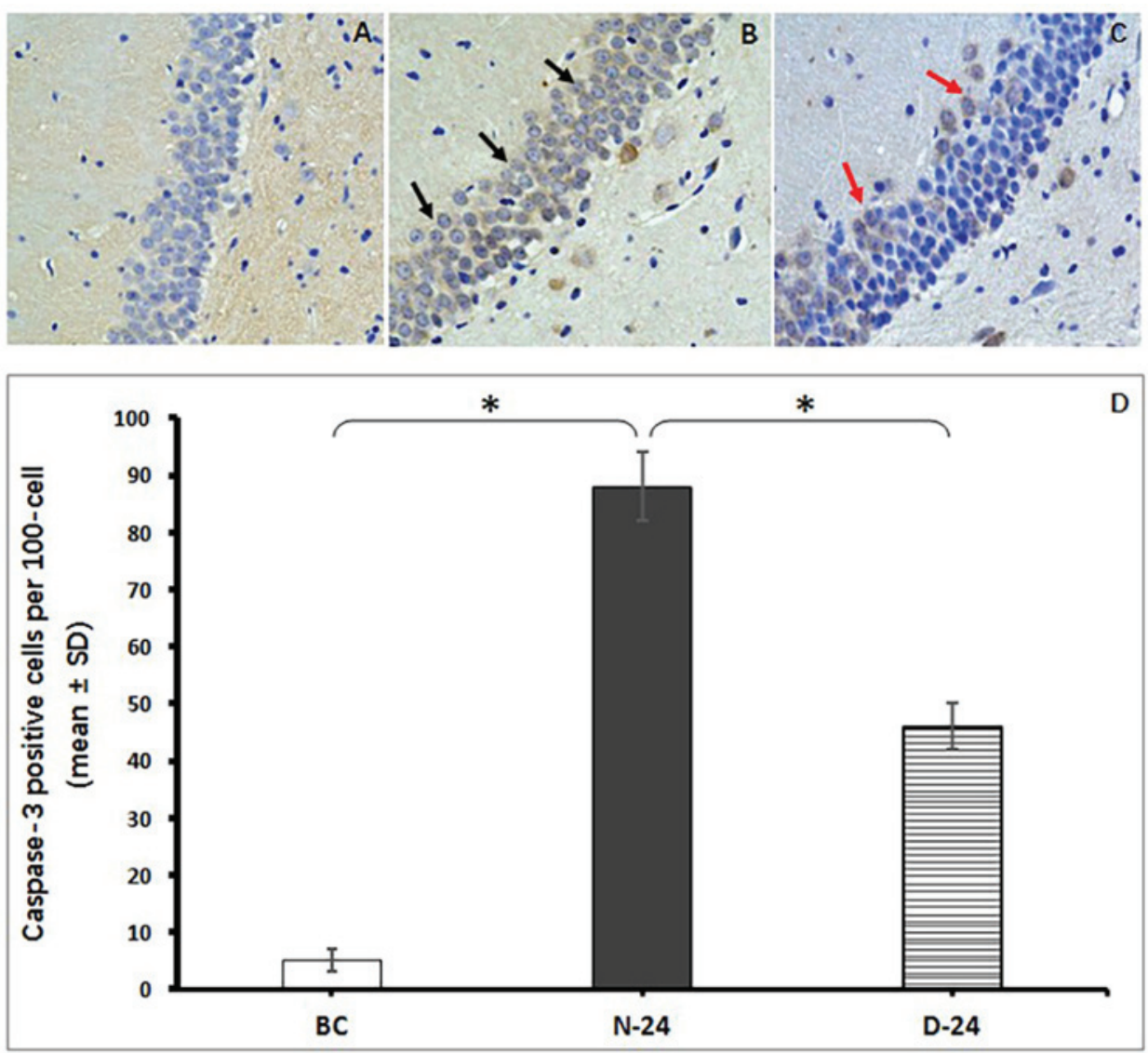

Figure 6. Comparison of Caspase-3 positive cells by immunohistochemical analysis. IHC techniques were used in the rat CA1 region of the hippocampus $24 \mathrm{~h}$ after ROSC, in the presence of normal saline or dexmedetomidine. (A-C) Brown globules indicate Caspase-3-positive expression, which is indicative of apoptosis as detected in (A) BC; (B) N-24, in which the brown globules were abundant and intensive (black arrows); and (C) D-24, in which the brown globules were less prominent compared with the $\mathrm{N}$ group (red arrows). (D) Average number of Caspase-3-positive cells of each rat derived from 100 cells of four different fields of view (marked with mean \pm SD; magnification, $\mathrm{x} 200$ ). "P<0.05. BC, blank control group; D-24, dexmedetomidine group $24 \mathrm{~h}$ post-ROSC; $\mathrm{N}-24$, normal saline group $24 \mathrm{~h}$ post-ROSC; ROSC, return of spontaneous circulation.
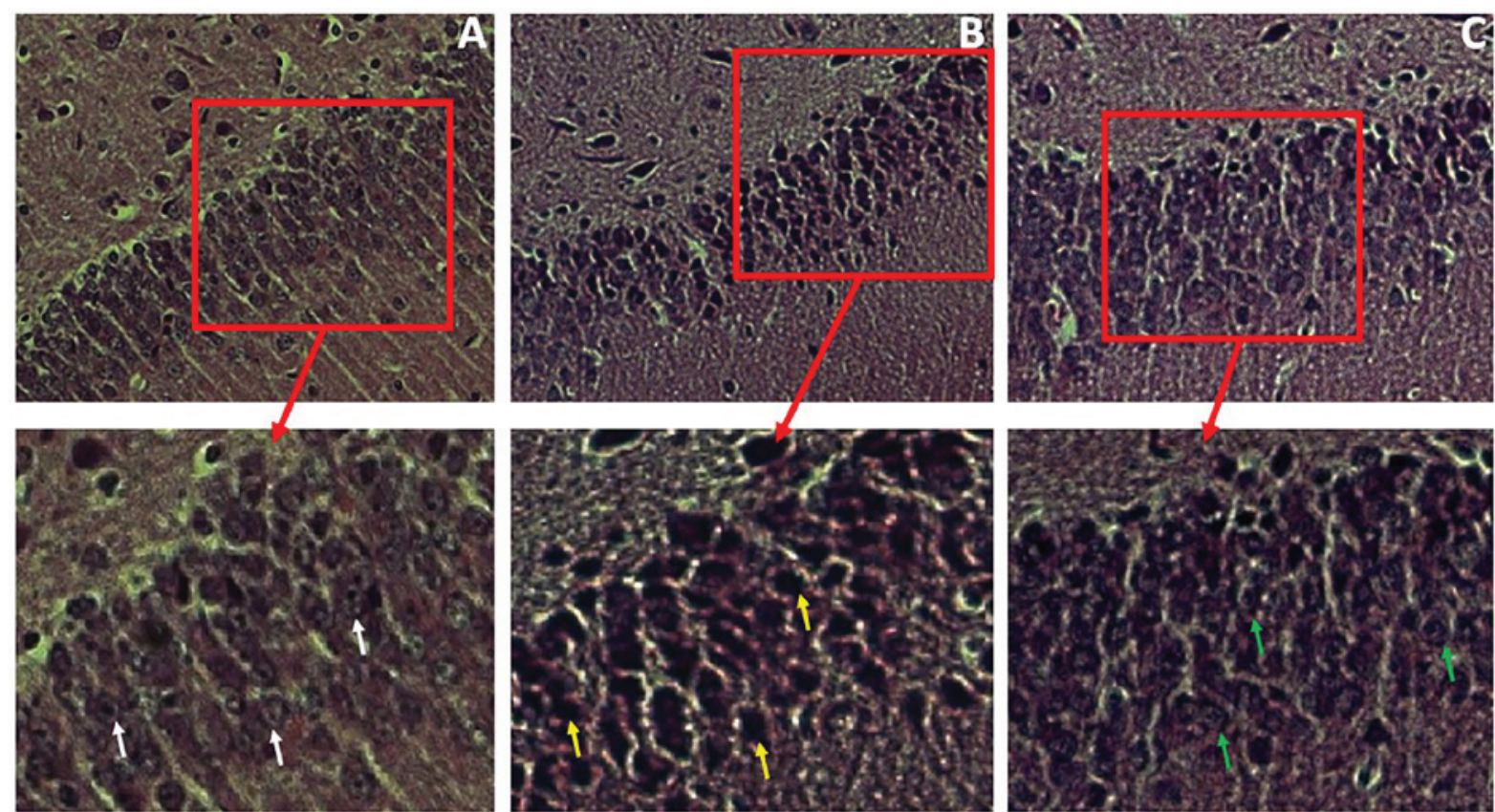

Figure 7. Hematoxylin and eosin staining of the rat CA1 region using light microscopy. Hippocampal tissue were isolated from rats $24 \mathrm{~h}$ following ROSC in the presence of normal saline or dexmedetomidine. (A) In the BC group, the shape and content of neurocytes were distinct (white arrows). (B) In the N-24 group, the shape of neurocytes was vague with visible shrinkage in the volume and content of neurocytes (yellow arrows). (C) In the D-24 group, the shape, volume and the organelles of neurocytes were more comparable (green arrows) to the BC group in image A. Magnification, x200; magnified areas are boxed in red. BC, blank control group; D-24, dexmedetomidine group $24 \mathrm{~h}$ post-ROSC; N-24, normal saline group $24 \mathrm{~h}$ post-ROSC; ROSC, return of spontaneous circulation. 


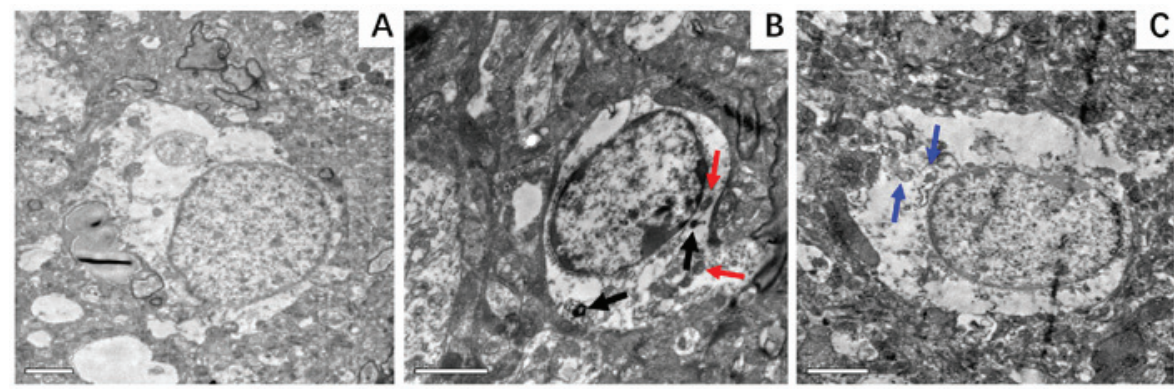

Figure 8. Representative electron micrographs of hippocampal neurocytes nuclei. Hippocampal tissue was isolated from the rat hippocampus at $24 \mathrm{~h}$ following ROSC. (A) BC group, demonstrating a normal nucleus with an intact nuclear membrane and smooth chromatin; magnification, $\mathrm{x} 8,000$. (B) N-24 group, displaying a markedly damaged nucleus with chromatin margination and condensed nucleoplasm, with autophagosomes (black arrows) and heteromorphic mitochondria (red arrows) in the cytoplasm; magnification, x12,000. (C) D-24 group, displaying a slightly damaged nucleus with normal nuclear membrane, slightly damaged chromatin and mitochondria (blue arrows) in the cytoplasm; magnification, x10,000. BC, blank control group; D-24, dexmedetomidine group $24 \mathrm{~h}$ post-ROSC; $\mathrm{N}-24$, normal saline group $24 \mathrm{~h}$ post-ROSC; ROSC, return of spontaneous circulation.

injury. A number of previous studies have demonstrated that Dex can alleviate I/R-induced neurocyte injury either by an intrinsic Bax/Caspase pathway (38) or by inhibiting neuronal autophagy through the upregulation of hypoxia-inducible factor $1 \alpha$ expression (39). Bcl-2 binding to Beclin1 reduces its capacity to activate autophagy without losing its own anti-apoptotic characteristics (40). The results of the present study support the hypothesis that neuronal apoptosis and excessive autophagy may be part of the pathophysiological process in $\mathrm{I} / \mathrm{R}$ injury following $\mathrm{CA}$, a process that can at least in part be alleviated by Dex.

The present study carries a number of limitations that needs to be addressed in future investigations. The dosage and method of Dex administration would need to be optimized and the mechanism of Dex-induced hypothermia would require further elucidation; both of which can be resolved by in vitro studies. Furthermore, the background luminance of pathological sections was inadequate, making images appear dark in color. In addition, the present study was performed on a rat model in the absence of underlying cardiac-cerebrovascular disease, hampering applicability to human patients.

In the present study, it was found that $\mathrm{I} / \mathrm{R}$ injury activated Bax, Caspase- 3 and Beclin1 expression, which indicated that apoptosis and excessive autophagy may be the main pathological process post-ROSC. The administration of Dex reduced Bax, Caspase-3 and Beclin1 expression levels, induced Bcl-2 and HSP70 expression and hypothermia, and improved neurological function. Therefore, the activation of intracellular apoptosis and autophagy following $\mathrm{I} / \mathrm{R}$ contributes to neural injury, whereas Dex treatment may induce hypothermia, decrease cellular injury of neurons and improve neurological function.

In conclusion, CA-induced I/R can lead to apoptosis and excessive autophagy in hippocampal neurons. Dex treatment induced hypothermia, in addition to exerting anti-apoptotic and anti-autophagic effects to improve post-resuscitation neurological function after CA. The present study uncovered a potential new treatment strategy for CA recovery.

\section{Acknowledgements}

The authors would like to thank the Suzhou Health Commission (Jiangsu, China) for their financial support, the
Experimental Center of the Second Affiliated Hospital of Soochow University (Jiangsu, China) for their experimental guidance and the Experimental Center of Suzhou Municipal Hospital and pathology department of Suzhou Vocational Health College (Jiangsu, China) for their technical assistance.

\section{Funding}

The current study was supported by a grant from the Science and Technology Project 'Health of Science and Education' of Suzhou Province (grant no. KJXW2013026).

\section{Availability of data and materials}

All data generated or analyzed during this study are included in this published article.

\section{Authors' contributions}

JL proposed the current study and together with LJL, drafted the manuscript. JL and ZL performed the experiments. ZL collected and analyzed experimental data. SY and ZZ modified the manuscript. ZC performed histological and ultrastructural analysis. All authors read and approved the final manuscript for publication.

\section{Ethical approval and consent to participate}

The current study was performed according to the exact guidelines and protocols for animal care and use established by the Experimental Center of Suzhou Municipal Hospital (Animal Experiment Permit Number, 120410), and was approved by the Suzhou Municipal Hospital Ethic Committee (Ethical Review Number, KL901008).

\section{Patient consent for publication}

Not applicable.

\section{Competing interests}

The authors declare that they have no competing interests. 


\section{References}

1. Sanganalmath SK, Gopal P, Parker JR, Downs RK, Parker JC Jr and Dawn B: Global cerebral ischemia due to circulatory arrest: Insights into cellular pathophysiology and diagnostic modalities. Mol Cell Biochem 426: 111-127, 2017.

2. Girotra S, Chan PS and Bradley SM: Post-resuscitation care following out-of-hospital and in-hospital cardiac arrest. Heart 101: 1943-1949, 2015.

3. Ryu JA, Cho YH, Sung K, Choi SH, Yang JH, Choi JH, Lee DS and Yang JH: Predictors of neurological outcomes after successful extracorporeal cardiopulmonary resuscitation. BMC Anesthesiol 15: 26, 2015

4. Lu J, Qian HY, Liu LJ, Zhou BC, Xiao Y, Mao JN, An GY, Rui MZ, Wang T and Zhu CL: Mild hypothermia alleviates excessive autophagy and mitophagy in a rat model of asphyxial cardiac arrest. Neurol Sci 35: 1691-1619, 2014.

5. Lu J, Shen Y, Liu LJ, Qian HY and Zhu CL: Combining epinephrine and esmolol attenuates excessive autophagy and mitophagy in rat cardiomyocytes after cardiac arrest. J Cardiovasc Pharmacol 66: 449-456, 2015.

6. Yu ZH, Cai M, Xiang J, Zhang ZN, Zhang JS, Song XL, Zhang W, Bao J, Li WW and Cai DF: PI3K/Akt pathway contributes to neuroprotective effect of Tongxinluo against focal cerebral ischemia and reperfusion injury in rats. J Ethnopharmacol 181: $8-19,2016$

7. Lin JY, Zhang MW, Wang JG, Li H, Wei HY, Liu R, Dai G and Liao XX: Hydrogen sulfide improves neural function in rats following cardiopulmonary resuscitation. Exp Ther Med 11: 577-587, 2016

8. Ma Y, Chen C, Zhang S, Wang Q, Chen H, Dong Y, Zhang Z, Li Y, Niu Z, Zhu T, et al: RNase alleviates neurological dysfunction in mice undergoing cardiac arrest and cardiopulmonary resuscitation. Oncotarget 8: 53084-53099, 2017.

9. Zhang JC, Lu W, Xie XM, Pan H, Wu ZQ and Yang GT: Mild hypothermia attenuates post-resuscitation brain injury through a V-ATPase mechanism in a rat model of cardiac arrest. Genet Mol Res 15, 2016.

10. Zhou T, Lin H, Jiang L, Yu T, Zeng C, Liu J and Yang Z: Mild hypothermia protects hippocampal neurons from oxygen-glucose deprivation injury through inhibiting caspase-3 activation. Cryobiology 80: 55-61, 2018.

11. Rincon F: Targeted temperature management in brain injured patients. Neurosurg Clin N Am 29: 231-253, 2018.

12. Qin H, Tan W, Zhang Z, Bao L, Shen H, Wang F, Xu F and Wang Z: 15d-prostaglandin J2 protects cortical neurons against oxygen-glucose deprivation/reoxygenation injury: Involvement of inhibiting autophagy through upregulation of Bcl-2. Cell Mol Neurobiol 35: 303-312, 2015.

13. Wu X, Mao H, Liu J, Xu J, Cao J, Gu X and Cui G: Dynamic change of SGK expression and its role in neuron apoptosis after traumatic brain injury. Int J Clin Exp Pathol 6: 1282-1293, 2013.

14. Zhou X, Liu Y, Huang Y, Zhu S, Zhu J and Wang R: Hypertonic saline infusion suppresses apoptosis of hippocampal cells in a rat model of cardiopulmonary resuscitation. Sci Rep 7: 5783, 2017.

15. Rizos IK, Tsoporis JN, Toumpoulis IK, Salpeas V, Izhar S, Rigopoulos AG, Sakadakis EA and Parker TG: Antiapoptotic effect of $\beta 1$ blockers in ascending thoracic aortic smooth muscle cells: The role of HSP70 expression. J Cardiovasc Pharmacol 72: 86-96, 2018.

16. Choudhary GS, Al-Harbi S and Almasan A: Caspase-3 activation is a critical determinant of genotoxic stress-induced apoptosis Methods Mol Biol 1219: 1-9, 2015.

17. Liu Y, Phuong Anh Nguyen T, Chen M and Xie L: Tea polyphenols down-regulate JNK phosphorylation to inhibit neuron apoptosis in rats with cardiac arrest. Zhonghua Wei Zhong Bing Ji Jiu Yi Xue 29: 1122-1126, 2017 (In Chinese).

18. Klionsky DJ and Ohsumi Y: Vacuolar import of proteins and organelles from the cytoplasm. Annu Rev Cell Dev Biol 15: 1-32, 1999.

19. Shi R, Weng J, Zhao L, Li XM, Gao TM and Kong J: Excessive autophagy contributes to neuron death in cerebral ischemia. CNS Neurosci Ther 18: 250-1260, 2012.

20. Lu J, Shen Y, Qian HY, Liu LJ, Zhou BC, Xiao Y, Mao JN, An GY, Rui MZ, Wang T and Zhu CL: Effects of mild hypothermia on the ROS and expression of caspase- 3 mRNA and LC3 of hippocampus nerve cells in rats after cardiopulmonary resuscitation. World J Emerg Med 5: 298-305, 2014.
21. Gu Y, Chen T, Li G, Xu C, Xu Z, Zhang J, He K, Zheng L, Guan Z, $\mathrm{Su} X$, et al: Lower Beclin 1 downregulates HER2 expression to enhance tamoxifen sensitivity and predicts a favorable outcome for ER positive breast cancer. Oncotarget 8: 52156-52177, 2016.

22. Li L, You LS, Mao LP, Jin SH, Chen XH and Qian WB: Combing oncolytic adenovirus expressing Beclin-1 with chemotherapy agent doxorubicin synergistically enhances cytotoxicity in human CML cells in vitro. Acta Pharmacol Sin 39: 251-260, 2018.

23. Nikoletopoulou V, Markaki M, Palikaras K and Tavernarakis N: Crosstalk between apoptosis, necrosis and autophagy. Biochim Biophys Acta 1833: 3448-3459, 2013.

24. Afonso J and Reis F: Dexmedetomidine: Current role in anesthesia and intensive care. Rev Bras Anestesiol 62: 118-133, 2012

25. Chen X, Li L, Hu J, Zhang C, Pan Y, Tian D and Tang Z: Anti-inflammatory effect of dexmedetomidine combined with hypothermia on acute respiratory distress syndrome in rats. J Surg Res 216: 179-184, 2017.

26. Zakaria S, Kwong HJ, Sevransky JE, Williams MS and Chandra-Strobos N: The cardiovascular implications of sedatives in the cardiac intensive care unit. Eur Heart J Acute Cardiovasc Care 7: 671-683, 2018.

27. Yeda X, Shaoqing L, Yayi H, Bo Z, Huaxin W, Hong C and Zhongyuan X: Dexmedetomidine protects against renal ischemia and reperfusion injury by inhibiting the P38-MAPK/TXNIP signaling activation in streptozotocin induced diabetic rats. Acta Cir Bras 32: 429-439, 2017.

28. Geocadin RG, Ghodadra R, Kimura T, Lei H, Sherman DL, Hanley DF and Thakor NV: A novel quantitative EEG injury measure of global cerebral ischemia. Clin Neurophysiol 111: 1779-1787, 2000.

29. Shelton KT, Qu J, Bilotta F, Brown EN, Cudemus G, D'Alessandro DA, Deng H, DiBiasio A, Gitlin JA, Hahm EY, et al: Minimizing ICU neurological dysfunction with dexmedetomidine-induced sleep (MINDDS): Protocol for a randomised, double-blind, parallel-arm, placebo-controlled trial. BMJ Open 8: e020316, 2018.

30. Sato K, Kimura T, Nishikawa T, Tobe $Y$ and Masaki $Y$ : Neuroprotective effects of a combination of dexmedetomidine and hypothermia after incomplete cerebral ischemia in rats. Acta Anaesthesiol Scand 54: 377-382, 2010.

31. McAdams RM, McPherson RJ, Kapur R, Phillips B, Shen DD and Juul SE: Dexmedetomidine reduces cranial temperature in hypothermic neonatal rats. Pediatr Res 77: 772-778, 2015.

32. Lähdesmäki J, Sallinen J, MacDonald E, Sirviö J and Scheinin M: Alpha2-adrenergic drug effects on brain monoamines, locomotion and body temperature are largely abolished in mice lacking the alpha2A-adrenoceptor subtype. Neuropharmacology 44 : 882-892, 2003

33. Callaway CW, Elmer J, Guyette FX, Molyneaux BJ, Anderson KB Empey PE, Gerstel SJ, Holquist K, Repine MJ and Rittenberger JC: Dexmedetomidine reduces shivering during mild hypothermia in waking subjects. PLoS One 10: e0129709, 2015.

34. Gifford JR, Ives SJ, Park SY, Andtbacka RH, Hyngstrom JR, Mueller MT, Treiman GS, Ward C, Trinity JD and Richardson RS: $\alpha 1$ - and $\alpha 2$-adrenergic responsiveness in human skeletal muscle feed arteries: The role of TRPV ion channels in heat-induced sympatholysis. Am J Physiol Heart Circ Physiol 307: H1288-H1297, 2014.

35. Behmenburg F, Pickert E, Mathes A, Heinen A, Hollmann MW, Huhn R and Berger MM: The cardioprotective effect of dexmedetomidine in rats is dose-dependent and mediated by $\mathrm{BKCa}$ channels. J Cardiovasc Pharmacol 69: 228-235, 2017.

36. Ma Y, Yu XY and Wang Y: Dose-related effects of dexmedetomidine on immunomodulation and mortality to septic shock in rats. World J Emerg Med 9: 56-63, 2018.

37. Zhang MH, Zhou XM, Cui JZ, Wang KJ, Feng Y and Zhang HA: Neuroprotective effects of dexmedetomidine on traumatic brain injury: Involvement of neuronal apoptosis and HSP70 expression. Mol Med Rep 17: 8079-8086, 2018.

38. Wu GJ, Chen JT, Tsai HC, Chen TL, Liu SH and Chen RM: Protection of dexmedetomidine against ischemia/reperfusion-induced apoptotic insults to neuronal cells occurs via an intrinsic mitochondria-dependent pathway. J Cell Biochem 118: 2635-2644, 2017.

39. Luo C, Ouyang MW, Fang YY, Li SJ, Zhou Q, Fan J, Qin ZS and Tao T: Dexmedetomidine protects mouse brain from ischemia-reperfusion injury via inhibiting neuronal autophagy through up-regulating HIF-1 $\alpha$. Front Cell Neurosci 11: 197, 2017.

40. Ciechomska IA, Goemans GC, Skepper JN and Tolkovsky AM: Bcl-2 complexed with Beclin-1 maintains full anti-apoptotic function. Oncogene 28: 2128-2141, 2009. 